\title{
Absence of mutations in NR2EI and SNX3 in five patients with MMEP (microcephaly, microphthalmia, ectrodactyly, and prognathism) and related phenotypes
}

\author{
Ravinesh A Kumar ${ }^{1}$, David B Everman ${ }^{2}$, Chad T Morgan², Anne Slavotinek ${ }^{3}$, \\ Charles E Schwartz ${ }^{2}$ and Elizabeth M Simpson*1
}

Address: ${ }^{1}$ Centre for Molecular Medicine and Therapeutics, Child \& Family Research Institute, Department of Medical Genetics, University of British Columbia, 950 West 28th Ave, Vancouver, V5Z 4H4, Canada, ${ }^{2}$ Center for Molecular Studies, J.C. Self Research Institute, Greenwood Genetic Center. One Gregor Mendel Circle, Greenwood, South Carolina, 29646, USA and ${ }^{3}$ Department of Pediatrics, Division of Medical Genetics, University of California, Box 0748, 533 Parnassus St., San Francisco, California, 94143-0748, USA

Email: Ravinesh A Kumar - rakumar@cmmt.ubc.ca; David B Everman - deverman@ggc.org; Chad T Morgan - morgan114@charter.net; Anne Slavotinek - slavotia@peds.ucsf.edu; Charles E Schwartz - ceschwartz@ggc.org; Elizabeth M Simpson* - simpson@cmmt.ubc.ca

* Corresponding author

Published: 26 July 2007

BMC Medical Genetics 2007, 8:48 doi:10.1 I86/147|-2350-8-48

This article is available from: http://www.biomedcentral.com/I47I-2350/8/48

(C) 2007 Kumar et al; licensee BioMed Central Ltd.

This is an Open Access article distributed under the terms of the Creative Commons Attribution License (http://creativecommons.org/licenses/by/2.0), which permits unrestricted use, distribution, and reproduction in any medium, provided the original work is properly cited.

\begin{abstract}
Background: A disruption of sorting nexin 3 (SNX3) on 6q2I was previously reported in a patient with MMEP (microcephaly, microphthalmia, ectrodactyly, and prognathism) and $t(6 ; \mid 3)(q 2 I ; q \mid 2$ ) but no SNX3 mutations were identified in another sporadic case of MMEP, suggesting involvement of another gene. In this work, SNX3 was sequenced in three patients not previously studied for this gene. In addition, we test the hypothesis that mutations in the neighbouring gene NR2EI may underlie MMEP and related phenotypes.
\end{abstract}

Methods: Mutation screening was performed in five patients: the $t(6 ; 13)(q 21 ; q 12)$ MMEP patient, three additional patients with possible MMEP or a related phenotype, and one patient with oligodactyly, ulnar aplasia, and a $t(6 ; 7)(q 21 ; q 31.2)$ translocation. We used sequencing to exclude SNX3 coding mutations in three patients not previously studied for this gene. To test the hypothesis that mutations in NR2EI may contribute to MMEP or related phenotypes, we sequenced the entire coding region, complete $5^{\prime}$ and $3^{\prime}$ untranslated regions, consensus splice-sites, and evolutionarily conserved regions including core and proximal promoter in all five patients. Twohundred and fifty control subjects were genotyped for any candidate mutation.

Results: We did not detect any synonymous nor nonsynonymous coding mutations of NR2EI or SNX3. In one patient with possible MMEP, we identified a candidate regulatory mutation that has been reported previously in a patient with microcephaly but was not found in 250 control subjects examined here.

Conclusion: Our results do not support involvement of coding mutations in NR2EI or SNX3 in MMEP or related phenotypes; however, we cannot exclude the possibility that regulatory NR2EI or SNX3 mutations or deletions at this locus may underlie abnormal human cortical development in some patients. 


\section{Background}

The MMEP phenotype and EEC syndrome represent syndromic forms of split-hand/foot malformation (SHFM), which occurs either as an isolated malformation or as a feature of many other conditions with overlapping clinical findings [1]. SHFM can be caused by chromosome rearrangements or single gene mutations and is a genetically heterogeneous malformation with multiple gene loci having been identified [1].

Sorting nexin 3 (SNX3) is ubiquitously expressed and belongs to the sorting nexin family, which is involved in intracellular protein trafficking [2]. Previously, SNX3 was found to be disrupted in its 3rd intron by a de novo balanced translocation $\mathrm{t}(6 ; 13)(\mathrm{q} 21 ; \mathrm{q} 12)$ in a patient with MMEP (microcephaly, microphthalmia, ectrodactyly, and prognathism) and severe mental retardation [3]. Thus, SNX3 was initially proposed as a reasonable candidate for MMEP in this patient, who did not harbor any SNX3 mutations on the normal chromosome [3]. However, mutations involving SNX3 were not identified in a sporadic case with possible MMEP and normal karyotype $[3,4]$. Thus, mutations in a gene close to SNX3 may contribute to MMEP and related phenotypes.

Nuclear receptor 2E1 (NR2E1; previously, TLX [MIM 603849]) is the closest gene to SNX3 (Figure 1) and therefore represents a strong positional candidate that may contribute to the brain phenotype of MMEP. NR2E1 is also a strong functional candidate, given that mice deleted for Nr2e1 present with a complex MMEP-related phenotype that includes forebrain hypoplasia, eye abnormalities, and cognitive impairment [5-7], which is consistent with the brain and eye expression pattern of this gene $[8,9]$. Further evidence is supported by the possibility that the der(6) breakpoint in the $t(6 ; 13)(q 21 ; q 12)$ patient could have created a position effect that altered the expression of NR2E1 resulting in the MMEP phenotype (Figure 1) [3]. Such a hypothesis, however, is difficult to test in light of the predominantly brain-specific transcription of NR2E1 $[8,10]$ and lack of suitable patient material.

We propose that a gene(s) near the 6q21 translocation may underlie MMEP and related phenotypes in some patients. Here, we test the hypothesis that patients with MMEP or a related phenotype may harbor mutations in NR2E1 and/or SNX3. To test this hypothesis, we sequenced the entire NR2E1 coding region, consensus splice-site regions, complete 5' and $3^{\prime}$ untranslated regions and evolutionarily conserved elements including core and proximal promoter in one patient with MMEP, three patients with possible MMEP or related phenotypes, and one patient with oligodactyly, ulnar aplasia, and a $\mathrm{t}(6 ; 7)(\mathrm{q} 21 ; \mathrm{q} 31.2)$ translocation [11]. We also sequenced

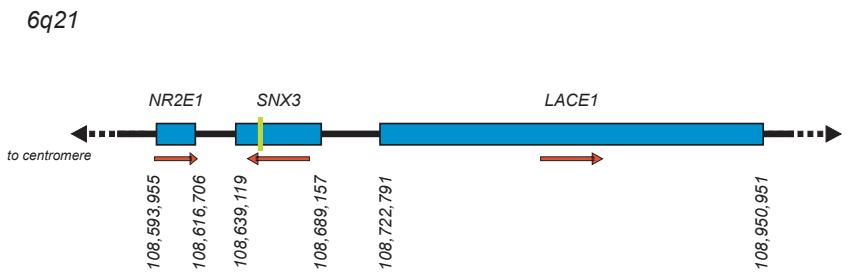

\section{Figure I}

Schematic drawing of $6 q 21$ demonstrates relative positions of genes flanking SNX3. Green bar indicates location of breakpoint within intron 3 of SNX3 in patient with $\mathrm{t}(6 ; 13)(\mathrm{q} 2|; q| 2)$ translocation [3]. Red arrows indicate direction of transcription. Genetic locations of transcriptional start and end sites are from RefSeq genomic assembly NC_000006.I0.

the complete coding regions of SNX3 in three of five patients not previously examined for this gene.

\section{Methods \\ Human subjects}

Approval for this study was obtained from The University of British Columbia and Child \& Family Research Institute. The research followed Canada's Tri-Council Statement on 'Ethical Conduct for Research Involving Humans'. Approval was also obtained through the Institutional Review Board of Self Regional Healthcare (Greenwood, SC). Consent from all patients was obtained for research purposes. Patients were ascertained and examined from five centers: The University of the Witwaterstrand, Johannesburg; The University of Cape Town, South Africa; Cedars-Sinai Medical Center, Los Angeles; Universita Cattolica, Rome; and The University of California, San Francisco. Patients were referred to the Greenwood Genetic Center for molecular research studies on split-hand/foot malformation. Controls were ascertained from the Greenwood Genetic Center.

All cases were sporadic and born to non-consanguineous parents. Patient 1 is the original patient with MMEP and $\mathrm{t}(6 ; 13)(\mathrm{q} 21 ; \mathrm{q} 12)$ described previously by Viljoen and Smart [12]. This is a 44-year-old Caucasian female with severe mental retardation, congenital microphthalmia causing complete blindness, central cleft lip and palate, ectrodactyly with absence of toes 2-4 on both feet, fingerlike thumbs, and a broad, prominent jaw.

Patients 2-4 had normal blood chromosome analyses and were included in this study on the basis of having clinical features that significantly overlapped those of the MMEP phenotype. Patient 2 was felt to have possible MMEP versus an unusual variant of the ectrodactyly-ectodermal dysplasia-clefting (EEC) syndrome and has not 
been described previously. She is a Hispanic female with congenital microcephaly, bilateral microphthalmia, colobomas of the right iris and retina, left chorioretinal coloboma, left lacrimal duct stenosis, unilateral cleft lip and palate, dysplastic ears, soft tissue syndactyly of fingers 34 on the right hand, ectrodactyly of the left foot with a hallux and two digital rays, unilateral mixed versus sensorineural hearing loss requiring a hearing aid, sparse scalp hair and eyebrows, and narrow, deep-set nails. She had a prolonged hospitalization after birth and required gastrostomy tube placement. She had no evidence of a TP73L (previously, TP63, P63) mutation, which is known to be involved in split hand/foot malformation [13].

Patients 3 and 4 were felt to have an unusual variant of the EEC syndrome. A brief summary of their clinical findings was reported previously [13]. Patient 3 is a 10-year-old Caucasian female with microcephaly, bilateral iris colobomas, microphthalmia with significant vision impairment, bilateral ectrodactyly of the hands and feet, unilateral cleft lip, and patchy alopecia of the scalp hair. She required gastrostomy feedings until age 9 due to ongoing problems with feeding, failure to thrive, and severe gastroesophageal reflux. A gastric emptying study revealed delayed emptying with a non-functioning section of the stomach and reverse peristalsis, which resolved with gastric Botox therapy. She has experienced significant dental problems due to ectodermal dysplasia. She attends a regular school program with visual assistance. Patient 4 is an African-American female seen at 3 months of age with congenital microcephaly, congenitally sealed eyelids with small to absent globes, ectrodactyly of the hands and feet, a notch in the upper lip resembling a mild midline cleft, absent scalp hair, underdeveloped eyelashes and eyebrows, underdeveloped nails, minor differences in ear shape, unilateral hearing loss, pelvic kidney, anteriorly placed anus, and tethered spinal cord. Both patients had no evidence of a TP73L mutation [13].

Patient 5 was included in the study on the basis of having a de novo chromosome translocation involving the $6 \mathrm{q} 21$ region $(\mathrm{t}(6 ; 7)(\mathrm{q} 21 ; \mathrm{q} 31.2))$ and congenital ulnar ray aplasia. His findings were described previously by Gurrieri et al. [11]. He is a Caucasian male evaluated in the newborn period with congenitally bowed radii, absent ulnae, absence of fingers 3-5 on the right hand, syndactyly of fingers 2-3 and absence of fingers 4-5 on the left hand, and a cyst of the septum pellucidum. He did not have microcephaly, ocular abnormalities, or other features of the MMEP phenotype. We cannot exclude the possibility that breakage at 7q31 may disrupt a gene(s) involved in MMEP.

\section{DNA amplification and sequencing}

Sequencing was used to screen for SNX3 coding mutations in all 4 exons as previously described [3]. We sequenced genomic NR2E1 using 20 PCR amplicons that covered the coding regions (1,146 bp), complete 5 ' and 3' UTRs (1,973 bp), exon-flanking regions including consensus splice-sites (1,719 bp), and evolutionarily conserved regions including the core and proximal promoter $(1,528$ bp). Polymerase chain reactions (PCR) and sequencing were performed as previously described [14]. Sequences were visually inspected and scored blindly by at least two individuals using either Consed [15] or Sequencher (Gene Codes, Ann Arbor, MI). Every variant identified was confirmed by repeating the PCR and sequencing process. Confirmation of the g.21502T>C change in patient 2 and genotyping of unaffected parents and 250 controls without MMEP or related phenotypes was performed by restriction enzyme digestion of a 505base pair PCR product using BsmBI. Primers and PCR conditions were as previously reported for 3' UTRb [14]. The alteration created an additional BsmBI site, producing bands of 505, 278, and 227 base pairs in the heterozygous state.

\section{Results and discussion Absence of SNX3 coding mutations in MMEP or related phenotypes}

To exclude the role of coding mutations in SNX3 in MMEP or related phenotypes, we sequenced all four coding exons in all patients not previously studied for this gene. No mutations were detected. The absence of coding mutations in SNX3 supports the role of other loci, such as NR2E1, in these disorders. We cannot, however, exclude the role of regulatory $S N X 3$ mutations given that promoter and UTRs were not examined.

\section{Absence of NR2EI coding mutations in MMEP or related phenotypes}

To determine whether patients with MMEP or related phenotypes harbor NR2E1 mutations, we sequenced the complete coding region, complete 5' and 3' UTR, consensus splice-sites, and evolutionarily conserved regions including the core and proximal promoter in all five patients. We generated approximately $33.5 \mathrm{~kb}$ of sequence data. We did not detect any synonymous nor nonsynonymous coding mutations.

We identified one candidate regulatory mutation in the 3' UTR, g. 21502T>C, in patient 2, that was not found in dbSNP (Build 127) [16]. We confirmed this variant by resequencing both strands of DNA. The variant was not predicted to alter binding of neural transcription factors [14]. We genotyped g.21502T>C in the unaffected parents and identified the candidate regulatory mutation in the mother but not the father (Figure 2). In addition, we did 
not find g.21502T>C in 500 chromosomes from approximately 250 controls without MMEP or related phenotypes. Interestingly, we previously reported the g.21502T>C candidate regulatory mutation (in addition to two other mutations) in a patient with microcephaly and the unaffected father but not in 344 control chromosomes nor in 188 ethnically-diverse chromosomes [14]. Thus, g.21502T>C has so far been identified only in families that present with microcephaly but not in 1032 control chromosomes from subjects that do not present with microcephaly.

One possibility is that the g. $21502 \mathrm{~T}>\mathrm{C}$ is an innocuous substitution that does not contribute to disease but rather represents a rare variant in the general population. An alternative interpretation is that heterozygous NR2E1 mutations may contribute to disease, which is supported by studies in mice heterozygous for $\mathrm{Nr} 2 e 1$ deletions that show premature cortical neurogenesis early in development [17], thereby suggesting dosage sensitivity for NR2E1. The presence of g.21502T>C in an unaffected parent may be due to incomplete penetrance of this variant. Alternatively, g.21502T>C may interact with another susceptibility variant arising de novo in the patient or inherited from the non-g.21502T>C-transmitting parent. Such a mechanism is supported by genetic studies in mice in which double heterozygous mutations at $\mathrm{Nr} 2 e 1$ and Pax6 are shown to enhance cortical phenotypes [18]. It is

A

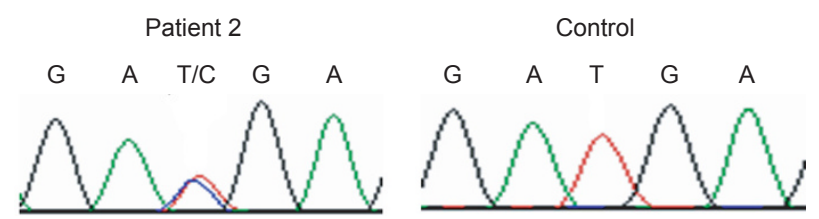

B

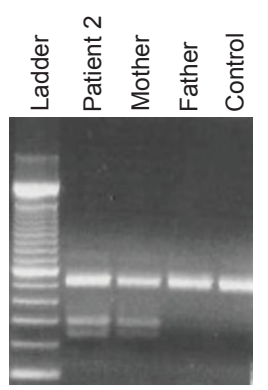

Figure 2

Genotyping NR2E I candidate regulatory mutation in patient 2 and family members. A) DNA sequencing detects g. $21502 T>C$ in the $3^{\prime}$ UTR of patient 2 but not in unrelated controls. B) Restriction digest detects g.2 I 502T>C in the unaffected mother but not in the unaffected father nor in an unrelated control. important to note that the clinical phenotypes of the patients in this study may be attributable to different genetic mechanisms affecting similar developmental pathways.

\section{Conclusion}

MMEP and related phenotypes represent a spectrum of heterogeneous conditions for which multiple loci may be involved, including NR2E1 and SNX3 on Chromosome 6q21-22 [11,12]. The present study does not support involvement of NR2E1 or SNX3 coding mutations in MMEP or related phenotypes. However, we cannot exclude the possibility that regulatory NR2E1 or SNX3 mutations, such as g.21502T $>C$ of NR2E1, may underlie abnormal human cortical development in some families. In addition, we cannot exclude the possibility that deletions at NR2E1 or SNX3 may underlie MMEP, given that sequencing is unable to distinguish between homozygosity across loci versus large deletions. The lack of obvious mutations in NR2E1 and SNX3 contribute to the genetic complexity underlying this heterogeneous syndrome. Follow-up studies of other positional candidates such as LACE1, would be a next logical undertaking.

\section{Competing interests}

The author(s) declare that they have no competing interests.

\section{Authors' contributions}

RAK conducted the NR2E1 molecular genetic studies, performed data analysis, and wrote the manuscript. DBE, AS, and CES collected families and provided DNA. CTM carried out the SNX3 sequencing and contributed to NR2E1 genotyping. EMS initiated the study and finalized the analyses as well as the paper. All authors read and approved the final manuscript.

\section{Acknowledgements}

The authors acknowledge the patients and families who donated their time and blood samples. We thank Dr. Denis L. Viljoen (University of the Witwaterstrand, Johannesburg), Dr. Ronald Smart (University of Cape Town, South Africa), Dr. John M. Graham, Jr. (Cedars-Sinai Medical Center, Los Angeles), and Dr. Fiorella Gurrieri (Universita Cattolica, Rome) for providing us with patient samples. We also thank Tonya Moss for assistance in sequence analyses of SNX3. The authors are grateful to Brett $\mathrm{S}$. Abrahams and Tracey D. Weir (Centre for Molecular Medicine and Therapeutics, Canada) for insight and helpful comments on the manuscript. This work was supported in part by grants from Jack and Doris Brown Foundation and British Columbia Institute for Children's \& Women's Health (to RAK); Shriners Hospitals for Children, Grant \#85I0 (to DBE); South Carolina Department of Disabilities and Special Needs (SCDDSN) (to CES); and Canadian Institutes for Health Research (CIHR), CIHR Research and Development, and Canada Research Chair in Genetics and Behaviour (to EMS). 


\section{References}

I. Duijf PH, van Bokhoven H, Brunner HG: Pathogenesis of splithand/split-foot malformation. Hum Mol Genet 2003, I 2 Spec No I:R5I-60.

2. Xu $Y$, Hortsman $H$, Seet $L$, Wong $\mathrm{SH}$, Hong W: SNX3 regulates endosomal function through its PX-domain-mediated interaction with PtdIns(3)P. Nature cell biology 200I, 3(7):658-666.

3. Vervoort VS, Viljoen D, Smart R, Suthers G, DuPont BR, Abbott A, Schwartz CE: Sorting nexin 3 (SNX3) is disrupted in a patient with a translocation $t(6 ; 13)(q 2 I ; q I 2)$ and microcephaly, microphthalmia, ectrodactyly, prognathism (MMEP) phenotype. Journal of medical genetics 2002, 39( I 2):893-899.

4. Suthers G, Morris L: A second case of microcephaly, microphthalmia, ectrodactyly (split-foot) and prognathism (MMEP). Clinical dysmorphology 1996, 5(I):77-79.

5. Young KA, Berry ML, Mahaffey CL, Saionz JR, Hawes NL, Chang B, Zheng QY, Smith RS, Bronson RT, Nelson RJ, Simpson EM: Fierce: a new mouse deletion of $\mathrm{Nr} 2 \mathrm{el}$; violent behaviour and ocular abnormalities are background-dependent. Behav Brain Res 2002, I32(2): | 45-I58.

6. Roy K, Thiels E, Monaghan AP: Loss of the tailless gene affects forebrain development and emotional behavior. Physiol Behav 2002, 77(4-5):595-600

7. Kumar RA, Chan KL, Wong AH, Little KQ, Rajcan-Separovic E, Abrahams BS, Simpson EM: Unexpected embryonic stem (ES) cell mutations represent a concern in gene targeting: lessons from "fierce" mice. Genesis 2004, 38(2):5I-57.

8. Jackson A, Panayiotidis P, Foroni L: The human homologue of the Drosophila tailless gene (TLX): Characterization and mapping to a region of common deletion in human lymphoid leukemia on chromosome 6q2 I. Genomics 1998, 50(I):34-43.

9. Monaghan AP, Grau E, Bock D, Schutz G: The mouse homolog of the orphan nuclear receptor tailless is expressed in the developing forebrain. Development 1995, I 2 I (3):839-853.

10. Nishimura M, Naito S, Yokoi T: Tissue-specific mRNA Expression Profiles of Human Nuclear Receptor Subfamilies. Drug Metab Pharmacokinet 2004, I 9(2): I35- I49.

II. Gurrieri F, Cammarata M, Avarello RM, Genuardi M, Pomponi MG Neri G, Giuffre L: Ulnar ray defect in an infant with a $6 q 21 ; 7 q 31.2$ translocation: further evidence for the existence of a limb defect gene in 6q2I. Am J Med Genet 1995, 55(3):315-318.

12. Viljoen DL, Smart R: Split-foot anomaly, microphthalmia, cleftlip and cleft-palate, and mental retardation associated with a chromosome 6;13 translocation. Clinical dysmorphology 1993, 2(3):274-277.

13. de Mollerat XJ, Everman DB, Morgan CT, Clarkson KB, Rogers RC, Colby RS, Aylsworth AS, Graham JM Jr., Stevenson RE, Schwartz CE: P63 mutations are not a major cause of non-syndromic split hand/foot malformation. Journal of medical genetics 2003, 40(I):55-6I.

14. Kumar RA, Leach S, Bonaguro R, Chen J, Yokom DW, Abrahams BS Seaver L, Schwartz CE, Dobyns W, Brooks-Wilson A, Simpson EM: Mutation and evolutionary analyses identify NR2EI-candidate-regulatory mutations in humans with severe cortical malformations. Genes Brain Behav 2006 in press.

15. Gordon D, Abajian C, Green P: Consed: a graphical tool for sequence finishing. Genome Res 1998, 8(3):195-202.

16. National Center for Biotechnology Information: dbSNP. [http:// www.ncbi.nlm.nih.gov/projects/SNP].

17. Roy K, Kuznicki K, Wu Q, Sun Z, Bock D, Schutz G, Vranich N, Monaghan AP: The TIx Gene Regulates the Timing of Neurogenesis in the Cortex. J Neurosci 2004, 24(38):8333-8345.

18. Stenman J, Yu RT, Evans RM, Campbell K: TIx and Pax6 co-operate genetically to establish the pallio-subpallial boundary in the embryonic mouse telencephalon. Development 2003, I30(6): I | |3-| | 22.

\section{Pre-publication history}

The pre-publication history for this paper can be accessed here:

\section{http://www.biomedcentral.com/1471-2350/8/48/prepub}

Publish with Bio Med Central and every scientist can read your work free of charge

"BioMed Central will be the most significant development for disseminating the results of biomedical research in our lifetime. "

Sir Paul Nurse, Cancer Research UK

Your research papers will be:

- available free of charge to the entire biomedical community

- peer reviewed and published immediately upon acceptance

- cited in PubMed and archived on PubMed Central

- yours - you keep the copyright 